\title{
Fortalecimiento de los proyectos educativos de las instituciones educativas oficiales del municipio de Santiago de Cali
}

\section{Strengthening of the educational projects of the official educational institutions of the municipality of santiago de Cali}

\author{
Milton Fernando Trujillo-Losada ${ }^{1}$ \\ María Cristina Hurtado-Zúñiga² \\ María Julieth Pérez-Paredes ${ }^{3}$
}

Recibido: octubre 04 de 2018 Aceptado: diciembre 03 de 2018

\section{Resumen}

El presente artículo expone los resultados de un proceso de investigación-acción, en el marco del convenio entre la Secretaría de Educación Municipal y el Instituto de Educación y Pedagogía de la Universidad del Valle, desarrollado con 24 Instituciones Educativas Oficiales, con el fin de reestructurar y actualizar su Proyecto Educativo Institucional, PEl. Para ello, se usaron dos estrategias en simultáneo en el periodo agostodiciembre de 2017: la primera, la formación docente sobre aspectos del PEI, la caracterización escolar e institucional y la redefinición del modelo pedagógico; y la segunda, el acompañamiento y seguimiento a cada institución. Como resultados se muestran los alcances y limitaciones de la experiencia, prioridades y recomendaciones sobre la necesidad de ampliar la mirada al contexto de la comunidad educativa, desde lo global a lo local, en coherencia con el momento histórico de construcción de cultura de paz en el país.

Palabras clave: proyecto educativo institucional, caracterización institucional, modelos pedagógicos.

\begin{abstract}
This article shows the results of an action research process, within the framework of the agreement between La Secretaria de Educación Municipal and the Instituto de Pedagogía de la Universidad del Valle, developed with 24 official educational institutions in order to restructure and update their Institutional Educational Project(IEP). For this, two strategies were used simultaneously between the months of August and December 2017. Firstly, a teacher training was required on aspects of the IEP, school and institutional characterization, and redefinition of the pedagogical model. Secondly, support and follow-up to each institution. The results show the scope and limitations of the experience, priorities and recommendations on the need to broaden the perspective of the educational community, from global to local, in coherence with the historical moment of construction of a culture of peace in the country.
\end{abstract}

Keywords: institutional educational project, institutional characterization, pedagogical models.

1 Licenciado en Filosofía, Magíster en Educación, Universidad del Valle, Cali, Colombia. E-mail: milton.trujillo@correounivalle.edu.co

2 Licenciada en Educación, Tecnología e Informática, Magíster en Administración de Empresas, Universidad del Valle, Cali, Colombia. E-mail: maria.cristina.hurtado@correounivalle.edu.co

3 Profesional en Estudios Políticos y Resolución de Conflictos, Universidad del Valle, Cali, Colombia. E-mail: maria.perez.paredes@ correounivalle.edu.co 


\section{Introducción}

El presente artículo se basa en la información recogida en el proceso de acompañamiento y seguimiento in situ, realizado por la Secretaría de Educación Municipal, SEM, y la Universidad del Valle - Instituto de Educación y Pedagogía, a 24 Instituciones Educativas Oficiales, IEO, en el marco del proyecto: fortalecimiento al Proyecto Educativo Institucional, PEl, para la ciudad de Cali. El propósito fue el de contribuir a la reflexión y acción en torno a la transformación del sistema educativo, replanteamiento de prácticas de planeación y gestión institucional y actualización de las prácticas pedagógicas de los docentes.

En el ámbito mundial, la UNESCO (2014), ha establecido cuatro pilares esenciales para llevar a cabo un proceso de educación que tenga como eje central el aprendizaje, a saber: primero, el aprender a conocer, con el que se invita a conocer el entorno y la cultura en los que será desarrollado. La acción educativa; segundo, el aprender a hacer, que busca no solo desarrollar competencias en los estudiantes, sino también con los profesores, que les permita accionar, innovar y articular los procesos de enseñanza y aprendizaje; tercero, el aprender a vivir, que busca ampliar la percepción y las diversas formas de convivencia en armonía y paz; $y$, cuarto, el aprender a ser, que fortalece no solo el aprendizaje de los estudiantes a través de la memoria y el razonamiento, sino también a través del sentido estético, las capacidades físicas, la aptitud para comunicar, entre otras habilidades del desarrollo humano.

En este sentido, se considera que al orientar el aprendizaje hacia la consolidación de los cuatro pilares mencionados, se puede contribuir al mejoramiento significativo de la calidad de vida, y del sistema educativo, solamente si los docentes empezamos a cambiar las prácticas y articulamos procesos de indagación a las rutinas de estudio, con dos propósitos esenciales: favorecer, primero, los procesos de formación, para que superen los niveles básicos de los procedimientos de búsque- da de información, y segundo, responder a las necesidades de la comunidad, generando posibles soluciones a los problemas planteados por la misma. Así, un sistema educativo que tiene a la base estos cuatro pilares, contribuye de manera más efectiva y pertinente a la formación de las nuevas generaciones, y responde a los nuevos contextos y entornos del siglo XXI, (Garcés-Pretel \& Ruiz-Cantillo, 2016).

En el contexto Latinoamericano, como afirman Marczak, Engelke, Bohl, y Saldarriaga Jiménez (2016), el capítulo de América Latina y el Caribe, del Banco Interamericcano de Desarrollo, BID, presenta un informe prospectivo en el que se evidencia que cada vez serán más difíciles las condiciones de ajuste a las infraestructuras y a la educación, teniendo en cuenta el avance en el envejecimiento en la población latinoamericana, lo que establece nuevos retos de fortalecimiento a los desarrollos educativos; lo anterior se convierte en aspecto clave para la gestión educativa y la planeación de los procesos educativos de la región. Esto no puede perder de vista el propósito que embarga la relación del sistema educativo con los contextos locales, pues los procesos de enseñanza y aprendizaje contextualizados deben llevar a una comprensión mayor del papel que las ciencias, sobre todo las humanas y sociales y, obviamente la capacidad del hombre, tienen para determinar los propósitos de sus propias acciones; por tanto, pensar en la relación educación y entorno parte de la concepción que la realidad no puede ser pensada sin un contexto social.

En este contexto se mueven las IEO del municipio de Santiago de Cali, Colombia, buscando las mejores estrategias para responder a las necesidades contemporáneas de educación, en sintonía con los propósitos misionales propios de la escuela. Una de las maneras que se han encontrado, y aprovechado en los últimos dos decenios de historia educativa local, para responder a la anterior coyuntura, ha estado determinada por el Ministerio de Educación Nacional de Colombia, MEN, y el Decreto 1860 de 1994, que permitió a cada Institu- 
ción Educativa la elaboración e implementación, con la participación de la comunidad educativa, de un Proyecto Educativo Institucional, PEl, que expresa documentalmente la forma como cada IEO ha decidido alcanzar los fines de la educación definidos por la Ley 115 (Congreso de la República, 1994). Se resaltan también las necesidades de los educandos, su comunidad local, regional y nacional, pero se adaptan a las condiciones sociales, económicas, culturales y ambientales de su entorno y cotidianidad.

Así, pensar en el mejoramiento de la calidad académica en nuestro país, exige la revisión del proceso de consolidación de los PEl, que lleva ya veintitrés años buscando fortalecer la mirada sobre el contexto y sus actores.

Abordamos entonces una experiencia en torno al proceso de reestructuración y actualización de los $\mathrm{PEI}$, conscientes de la dificultad de llevar a cabo un proceso que realmente cumpla con el criterio de participación activa de los diferentes actores de la comunidad educativa, necesario para brindar mayor legitimidad al proyecto orientador de los planes y acciones pedagógicas de la Institución Educativa. No podemos mantener la idea aislada y fragmentada del PEI como un mero instrumento de control o formalismo para el cumplimiento de la norma, pues más allá de un documento, el PEI recoge la dinámica de un grupo de personas, de una comunidad académica y de intelectuales, quienes vinculados a un proceso claro y continuo de seguimiento y evaluación constante, pueden actualizar y re-significar su PEI.

El propósito de la intervención fue contribuir a la actualización de la caracterización institucional, la contextualización y la definición de los modelos pedagógicos institucionales, concebidos como las bases para la reestructuración del PEI. El trabajo realizado fortaleció en las comunidades escolares los canales de participación y comunicación, pues la actualización de la caracterización contó con el aporte de las voces, sentires y percepciones de docentes, estudiantes y padres de familia, permitien- do robustecer también el escenario democrático de la escuela.

El trabajo de acompañamiento se hizo en 24 IEO a través de catorce sesiones, con la modalidad in situ. Este proceso se realizó en paralelo con el Diplomado de formación para los docentes y directivos docentes, estrategia complementaria para ahondar en algunos aspectos claves del PEI, para formar en la implementación de técnicas e instrumentos para actualizar la caracterización escolar en el marco del PEl, y para pensar la redefinición del modelo y enfoque pedagógico en coherencia con las necesidades institucionales y los resultados de la caracterización.

El presente artículo muestra las reflexiones del proceso anterior, producto de la experiencia, desglosadas en: alcances y limitaciones, prioridades $y$, finalmente, el compartir de algunas propuestas y recomendaciones para mantener la dinámica de revisión continua del PEl. Dichas reflexiones abordarán aspectos claves como la identidad institucional y la vigencia de los PEl; la necesidad de ampliar la mirada al contexto de la comunidad educativa, desde lo global a lo local; las necesidades de la formación con inclusión y consciente del momento histórico de construcción de cultura de paz en el país; así como reflexiones sobre el horizonte institucional y direccionamiento estratégico en los que son claves aspectos tales como la gestión del conocimiento y la cultura institucional.

\section{Marcos teórico y metodológico}

El Proyecto Educativo Institucional, PEl, es un instrumento de planeación estratégica para la organización de la prestación del servicio educativo (Rodriguez, 2015). Su elaboración es competencia de cada institución educativa, que, a partir de la autonomía que la ley le ha conferido, orienta, planea y ejecuta participativamente el proceso de enseñanza-aprendizaje. Es decir, define "su senda de acción y su horizonte educativo... de acuerdo con los cambios y necesidades del entorno y del contexto inmediato y lejano" (p. 100). Esto implica que 
la institución educativa debe asumir decisiones tanto de carácter administrativo como pedagógico y que no existe una única manera de lograrlo.

\subsection{Conceptos y metodologías en un PEI}

Duran, (1994) refiere que el Proyecto Educativo Institucional es una estrategia de cualificación de la educación que, a través de dos procesos básicos: el pedagógico-cultural y el organizativo-administrativo, tiene como propósito dotar de identidad y cohesión a la comunidad educativa en torno a la prospección, delimitación y materialización de "utopías", sueños y compromisos colectivos.

De este modo, y bajo el análisis guiado por el enfoque teórico interaccionista y procesual, se enfatiza en que el PEI no debe limitarse a la programación de actividades orientadas al logro de resultados acordes con unos objetivos preestablecidos, inamovibles y externos a los individuos, sino que debe observar y potenciar las interacciones que se establecen entre los sujetos que intervienen en la educación y que tienen como objetivo darle a esta un sentido. Todo porque el Proyecto Educativo Institucional "se estructura mediante el juego de las intersubjetividades que hace explícitas las intencionalidades, las concepciones y los enfoques que pretenden ponerse al servicio de la construcción del proyecto" (Duran, 1994). Así, se asume que el Proyecto Educativo Institucional debe plantearse en "la perspectiva de la educación como eje del desarrollo humano" $y$ "en torno a su incidencia en la reconfiguración de las relaciones interpersonales y sociales propias de los micro y macro entornos". Bajo una postura similar, (Lozano, 1999) plantea que:

El proyecto educativo se puede considerar como una acción cultural o acción instrumental. En principio, podríamos convenir que existen dos grandes tendencias sobre las concepciones del proyecto: una entiende el proyecto como estrategia de orden instrumental, como herramienta útil al servicio de la planificación técnica y al logro de la eficiencia interna de la productividad y/o del pro- ceso educativo y pedagógico, como plan de trabajo predeterminado y estático, orientado a la previsión de condiciones administrativas eficaces, en períodos rígidos establecidos. En el marco de tal concepción, un PEI buscaría fundamentalmente la eficiencia interna del sistema escolar, en términos de resultados referidos al rendimiento, deserción, cobertura, promoción, aprobación, mortalidad y retención escolar, entre otros.

La otra concepción considera el Proyecto como una estrategia cultural que centra su interés en el proyecto como proceso dinámico con sentido histórico, que permite articular en torno a él todo el quehacer educativo y destaca la importancia del mismo en cuanto posibilita a los protagonistas de la escuela en la educación como una alternativa para la transformación de la cultura educativa (Duran, 1994). En suma, se propone que, mediante la creación e implementación de los PEl, la escuela contribuya al proceso de formación de ciudadanos de cara a una revolución cultural de y desde la comunidad educativa, a partir de la promoción de la modificación de las mentalidades, los imaginarios y los comportamientos individuales y colectivos.

En cuanto a la metodología para trabajar los PEl, es posible identificar una suerte de consenso en que existen dos maneras de abordarlos, así como cualquier tipo de proyectos. "Una que, partiendo de diagnósticos del presente, identifica tendencias dominantes y busca alternativas de solución a los problemas existentes" (Durán, 1994, p.24), y que, por tanto, presta especial importancia a la formulación de la misión de la institución y a la verificación de su cumplimiento a través del desarrollo lineal de las siguientes fases: formulación de la misión, diagnóstico interno-externo, objetivos, plan, ejecución, seguimiento y ajuste, y evaluación. Lo anterior deja poco margen para la reformulación de los objetivos durante el proceso de ejecución.

Otra manera identificada de abordar los proyectos, es aquella que "toma como punto de referencia básico la utopía, asumida en constante oscilación entre el sueño y la acción" (Duran, 1994). En esta 
segunda manera cobra especial importancia el ejercicio histórico hermenéutico, es decir, la comprensión del presente (contraste de debilidades y fortalezas internas con amenazas y oportunidades externas) y del proceso mismo del PEl, en correspondencia a unas necesidades, intereses y exigencias de desarrollo y de cambio determinadas, para concretar el futuro a través del diseño.

\subsection{La mirada al contexto social y la relación con el entorno escolar}

El PEI es una propuesta de la institución educativa que se construye no solo en función de la comunidad educativa, o de su coherencia interna, sino también de su relevancia social: "pues este se concibe para generar cambios significativos en el campo del cual se ocupa, vale decir, para señalar alternativas que asuman críticamente lo existente y lo redimensionen al tenor de las necesidades humanas contemporáneas" (Duran, 1994).

Estas necesidades humanas contemporáneas apuntan a un cambio cultural, que supone"la apertura y el desarrollo de la democracia, la equidad, la participación y la concertación" (Lozano, 1999). La pertinencia del PEI se fundamenta en expresar la intencionalidad de aportar a la realización de las prioridades educativas y de desarrollo definidas a nivel regional y nacional, a la solución de los problemas del entorno escolar, al mejoramiento de la calidad de vida de los miembros de la comunidad educativa, a la convivencia y a la solución pacífica de los conflictos.

Algunas maneras de lograr este cometido son: emplear estrategias de articulación de la institución educativa con las expresiones culturales de su entorno, realizar investigaciones sobre el contexto social y cultural y sobre oferta y demanda educativa, así como establecer vínculos o relaciones con organizaciones sociales, como por ejemplo con los medios de comunicación, las organizaciones comunitarias y las agremiaciones económicas (Montes-Miranda, 2013; García-Amaya, Fernández-Morales \& Duarte, 2017).
Teniendo en cuenta lo anterior, consideramos que el diseño e implementación de la intervención, y su posterior análisis desde el enfoque de la Investigación Acción, materializó algunos preceptos normativos que rezan: "El Proyecto Educativo Institucional debe responder a situaciones y necesidades de los educandos, de la comunidad local, de la región y del país, ser concreto, factible y evaluable" (Art.73. Ley115/94), en coherencia con las siguientes premisas: a) EI PEI surge de la participación activa de todos los miembros de la comunidad educativa y es fruto de un consenso que ayuda a configurar la identidad de la IEO. b) Define la propuesta formativa de la Institución educativa, acorde a las políticas educativas nacionales, pero en coherencia con el conocimiento exhaustivo de la realidad institucional para asumir el contexto y las demandas locales, sin desconocer los conflictos y los problemas. c) Tiene como objetivo fundamental la formación integral de los estudiantes, el mejoramiento progresivo y sistemático de los resultados de aprendizaje y el perfeccionamiento de los procesos de enseñanza. d) Visualiza y anticipa la situación deseada, proponiéndose objetivos explícitos a lograr en el corto, mediano y largo plazo; delimitando las prioridades, los resultados deseados y las estrategias de acción para lograrlo. e) Establece instancias de evaluación acordes con la progresión en el logro de los objetivos, y articula la gestión escolar otorgando sentido y dirección pedagógica a los proyectos y programas de acción que allí se desarrollan.

\subsection{Metodología}

Se diseñaron dos estrategias para la implementación del proyecto de intervención desde la Investigación-Acción (Colmenares, 2012): la formación, a través de la modalidad de diplomado para docentes y directivos docentes, que permitió afianzar algunos referentes conceptuales y metodológicos para el fortalecimiento del PEl; y la estrategia de Acompañamiento y Seguimiento al Fortalecimiento del Proyecto Educativo Institucional, trabajo realizado in situ en cada una de las 24 IEO asignadas por la SEM. 
Por las limitaciones de tiempo y los requerimientos de necesidades contextuales, acudimos a la figura académica del diplomado como opción formativa para los docentes y directivos. La concepción básica de un diplomado como un curso largo preparado con la finalidad de afianzar conocimientos en áreas en las que las necesidades de la sociedad lo ameritan (Gómez-Resptrepo et al., 2014), nos permitió trabajar con la ventaja de la flexibilidad, y optamos por la modalidad de diplomado presencial con profundización en actividades virtuales, que sirvieron de estrategia de aprendizaje con la que los participantes acomodaron su tiempo y necesidades para profundizar y complementar algunos aspectos que se reflexionaban desde la presencialidad.

El nombre del diplomado, y su consecuente profundización, apuntó a fortalecer un proceso que las Instituciones Educativas deben seguir para el éxito de su funcionamiento. Decidimos Ilamarlo Fortalecimiento del Proyecto Educativo Institucional, desde una perspectiva resignificada de la Gestión Escolar, con el firme convencimiento de que este concepto incluye el conjunto de acciones y actividades relacionadas con la marcha cotidiana de la escuela: las de enseñanza y de aprendizaje; las administrativas; las que se realizan con la comunidad; las de organización y funcionamiento; las municipales, regionales y con otras escuelas; las prácticas de convivencia en la comunidad educativa y las articulaciones con otros proyectos o planes de mejoramiento. Adicionalmente, implica pensar en la gestión de las actividades administrativas y financieras; la organización de los directivos, de los profesores y de los alumnos; la planificación y diseño de la enseñanza; la organización del calendario anual y los horarios; la determinación de las normas de convivencia; la relación del establecimiento con los padres, madres y acudientes, y con otros organismos externos a la comunidad educativa, entre otros asuntos que dinamizan la vida cotidiana de una institución educativa.

Fortalecer el PEI significa realizar todas las acciones o diligencias, externas e internas al establecimien- to, que posibiliten cumplir la Misión que la comunidad se propuso al diseñar o elaborar su Proyecto Educativo (Ramírez et al., 2000). Acuñamos la perspectiva de la Gestión porque se inscribe en la realización de diligencias conducentes al logro de un proyecto. Administrar, consiste en gobernar, dirigir, ordenar, disponer u organizar; la Gestión, por lo tanto, implica el conjunto de acciones que se llevan a cabo para concretar un proyecto, en este caso, el Proyecto Educativo Institucional.

Los contenidos que se propusieron para el desarrollo de las sesiones del diplomado partieron de la premisa que concibe que: el proceso de reflexión sobre la educación implica cambios profundos en los escenarios, modelos y metodologías de la enseñanza, en primera instancia. Esta reflexión se propuso porque se piensa que los enfoques clásicos, centrados en el aula y en la actividad del profesor, deben dar paso a una enseñanza dirigida a nuevos aprendizajes a través de la actividad autónoma del estudiante. Este diplomado, con el desarrollo de cada uno de sus módulos, ayudó en esta difícil tarea, facilitando las herramientas y orientaciones necesarias para planificar, implementar y evaluar los procesos de enseñanza y aprendizaje bajo este nuevo enfoque. En este sentido, en el primer eje reflexivo del diplomado, se proyectó el tratamiento de algunos temas que han sido problematizados, debatidos, analizados y criticados, pero que ofrecen diversas alternativas para comprender su aplicación a situaciones concretas de la educación actual; también ofreció un espacio para la reflexión en torno a distintos problemas claves del mundo contemporáneo, con lo que se pretendió despertar la conciencia crítica, analítica y reflexiva de los participantes.

Un segundo eje de reflexión permitió que los participantes reconocieran, desde la teoría y la práctica, las habilidades necesarias para realizar una revisión del PEl, proyectar los cambios y ajustes que se requirieran, y profundizar en las rutas de mejoramiento que una institución requiere para el alcance y materialización de su propósito y misión educativa. En esa medida, se emplearon refuerzos 
conceptuales y procedimentales desde los campos de la pedagogía y la didáctica, revisando a su vez, algunos modelos educativos (contextualizados en la modernidad y contemporaneidad), y sus implicaciones en los procesos de enseñanza y aprendizaje, con el fin de lograr un nivel de reflexión sobre la propia práctica docente y el sentido y las posibilidades de la inter y la transdisciplinariedad, entre el mundo de la cultura, la epistemología y la experiencia docente. Algunos de los referentes asumieron la perspectiva de los modelos pedagógicos bancarios, conductista, constructivista, humanistas, sociales, de corte convencional o clásico, hasta la revisión de propuestas más innovadoras o integradoras de tipo holístico o sistémico.

Un tercer eje reflexivo contempló una integración de saberes, haceres y sentires, en lo que los referentes teóricos proporcionados en el análisis y la construcción conceptual derivaron elementos metodológicos para enriquecer las prácticas concretas de planeación y diagnóstico educativo, a través de un ejercicio para la implementación de técnicas e instrumentos, lo que permitió actualizar la caracterización o diagnóstico escolar en el marco del PEl.

La segunda estrategia del proyecto se desarrolló teniendo en cuenta los conceptos de participación, aprendizaje colaborativo y el desarrollo y la gestión de conocimiento en sus prácticas, con las que se favoreció el desarrollo de conocimiento colectivo, como condición para la creación de estrategias que permitieran el diálogo abierto y asequible de toda la comunidad académica. Este trabajo realizado in situ, con el aporte de los directivos de la IEO, de algunos representantes de los docentes y con el acompañamiento de un profesional de la Universidad del Valle, desde el comienzo, incentivó la distribución y reconocimiento de roles, definición de una ruta de aprendizaje propio a desarrollar, propiciar el manejo adecuado de la información y contribuir con un lenguaje común para la generación conjunta de ideas, potenciando espacios de liderazgo colectivo que permitieron el alcance de los objetivos propuestos.
En este ambiente se logró dimensionar el papel del profesional de acompañamiento, que se convirtió en un colega que visitaba las instituciones con una nueva mirada, para ver los problemas que surgían alrededor del desarrollo del PEI, no con el ánimo de solucionarlos desde su visión, sino de apoyar y aportar ideas para la solución compartida de los mismos. Así, todos los participantes se vincularon de una manera natural a un diálogo de saberes, que contribuyó al análisis y búsqueda de soluciones a los problemas.

La estrategia de Acompañamiento y Seguimiento al proceso incentivó en los docentes participantes del diplomado la apropiación de los conceptos y metodología para la "multiplicación" de los saberes en sus lugares de labor. Este acompañamiento para la revisión y actualización del diagnóstico institucional y la redefinición del modelo pedagógico, concebidas como las dos bases para la reestructuración del $\mathrm{PEI}$, se desarrolló en 14 sesiones de trabajo in situ. Algunas de estas sesiones se orientaron para la implementación de técnicas e instrumentos para actualizar la caracterización o diagnóstico escolar en el marco del PEl; otras, para redefinir del modelo y enfoque pedagógico en coherencia con las necesidades institucionales y los resultados de su caracterización; y unas cuantas sesiones restantes contribuyeron a la organización de la información que permitió estructurar los productos del proceso: el PEl actualizado, la caracterización institucional adelantada, la reflexión sobre el modelo pedagógico institucional iniciada y la definición de un plan de acción para continuar con el proceso al finalizar la intervención.

\section{Resultados y discusión}

(...) Por lo mismo, nuestra educación conformista y represiva parece concebida para que los niños se adapten por la fuerza a un país que no fue pensado para ellos, en lugar de poner el país al alcance de ellos para que lo transformen y engrandezcan. Semejante despropósito restringe la creatividad y la intuición congénita y contraría la imaginación, la clarividencia precoz y la sabiduría del corazón, 
hasta que los niños olviden lo que sin duda saben de nacimiento: que la realidad no termina donde dicen los textos, que su concepción del mundo es más acorde con la naturaleza que la de los adultos y que la vida sería más larga y feliz si cada quien pudiera trabajar en lo que le gusta, y solo eso. (García-Márquez, 1994)

Con esta reflexión se pretende hacer una aproximación descriptiva de las apuestas conceptuales y metodológicas, que fueron exitosas en el proceso de ajuste, actualización y re significación del Proyecto Educativo Institucional en las 24 instituciones participantes del proyecto; tales estrategias fueron propuestas y desarrolladas por los rectores, directivos docentes, profesores (equipo gestor), o sugeridas por los profesionales de acompañamiento. Este apartado fue construido teniendo en cuenta los reportes del desarrollo del proyecto en cada una de las IEO, base del informe final entregado por parte del equipo de la Universidad del valle a la SEM (Trujillo, 2017).

Del mismo modo, es necesario señalar las limitaciones que conllevan este tipo de procesos, no para evidenciar una carga negativa en ellas, sino para pensar en posibilidades que las puedan contrarrestar: Estas limitaciones se refieren a las actividades empleadas que no fueron del todo exitosas, bien sea por falta de tiempo, recursos o capacidades técnicas y logísticas.

\subsection{Horizonte institucional y direccionamiento es-} tratégico

Este apartado se vislumbró desde las siguientes premisas para su actualización y necesidades vigentes, tales como la construcción de la paz, los desafíos del pos conflicto, la promoción de los derechos humanos, la democracia, la participación, la equidad, la inclusión, la formación política para toda la comunidad educativa, la protección del medio ambiente, entre otros. Así pues, se tuvo en cuenta lo siguiente:
La misión se configuró desde los siguientes aspectos: ¿Qué hago como institución? ¿Para quién lo hago? ¿Cómo lo hago? (atributos, ejemplo: calidad, oportunidad, etc.) ¿Dónde lo hago? el compromiso de la IE con la sociedad. La visión se configuró desde los siguientes aspectos: una visión de futuro, se planteó a un horizonte de 5 años, se alineó con la misión definida, fue una construcción positiva y motivadora para toda la comunidad educativa. En algunas IEO se encontró que la misión y visión han sido actualizadas en diferentes espacios académicos, como el Comité de Convivencia y la Semana Institucional, principalmente; sin embargo, estos avances significativos reposaban en los anaqueles de las instituciones, no se habían incorporado al PEI, siendo así, la labor fue rastrear, ubicar y actualizar estos apartados, tomando como referencia las construcciones colectivas; es decir, se editaron y actualizaron sin cambiar su esencia.

Los Valores y Principios de la IEO, se configuraron desde las siguientes premisas: se han desplegado a toda la comunidad educativa, son representativos para toda la comunidad educativa. En algunas IEO la elaboración de los valores y principios involucró a grupos masivos de estudiantes, mediante talleres, en los que manifestaban qué valores son importantes para la IEO y cómo se vivencian en la misma, seguidamente el equipo gestor clasifica, ordena y edita los insumos para una versión final.

Los diferentes elementos de la filosofía organizacional (misión, visión y principios) se configuraron desde los siguientes principios: divulgados y comprendidos por toda la comunidad educativa, se construyeron de manera participativa, apropiados por toda la IEO, incluyendo a cada una de sus sedes; los diferentes elementos son consistentes con la calidad, la paz, la equidad y la inclusión como orientaciones de desarrollo. 


\subsection{La caracterización institucional}

La caracterización institucional se realizó teniendo en cuenta tres grandes aspectos, de lo general a lo particular: caracterización del entorno, caracterización de la IEO y caracterización de la población atendida, y se desglosan así:

Caracterización del entorno: componente social, componente económico, componente infraestructura física, componente cultural, componente ambiental, componente político. Esta construcción fue hecha desde dos miradas; la primera desde la revisión documental de textos gubernamentales, estadísticos y técnicos; por otro lado, desde la mirada de sus actores, por lo que en algunas instituciones se realizaron talleres con padres de familia, en los que debían hacer un relato de las historias de sus barrios, desafíos y oportunidades en su sector. Estos relatos luego fueron clasificados y sistematizados para elaborar el documento final; en otras IEO, los profesores complementaron la información técnica con sus propios relatos, desde su quehacer, experiencia y reconocimiento de su entorno; para esto fue muy útil el apoyo de los profesores de mayor antigüedad en el territorio. Ejemplo de esta caracterización y su importante relación con las proyecciones de los procesos educativos, la ofrece Gutiérrez-Rodríguez (2018) al señalar que en Cali, la mayoría de facultades del sector educativo, corresponden:

(...) Con la práctica misma de la enseñanza... (Pues) Para los profesores cada una de sus clases representa un reto en el propósito de atraer la atención de sus estudiantes, independientemente del nivel o área de formación... (y) resulta aún más complejo ya que las características de una época tecnológica, con una sobreoferta de medios audiovisuales como la televisión, el cine e internet, hace de dichos estudiantes individuos curiosos y muy dinámicos, que difícilmente se adaptan a los modelos pedagógicos tradicionales. (p. 280)
Razón por la cual la caracterización debe ser un ejercicio más cuidadoso y pertinente para la definición de la ruta institucional que forme adecuadamente a los estudiantes.

Caracterización de la IEO: componente formas organizativas y administrativas, componente infraestructura física y tecnológica, componente relaciones con el entorno, componente gobierno escolar, componente cultura institucional, componente clima escolar. Este fue uno de los componentes más satisfactorios de construir, dado que varios profesores aprovecharon para tener un acercamiento a las demás sedes que no conocían; se hicieron recorridos, conversaciones con los colegas de las sedes, y toma de registro fotográfico para caracterizar la infraestructura física y tecnológica; en la mayor parte de las IEO se conformaron comisiones de trabajo para caracterizar la cultura institucional y el clima escolar. Se encontraron en algunos casos dificultades en las relaciones interpersonales entre pares, maestro - directivo, directivo directivo o maestro - estudiante. Este espacio fue una especie de desahogo muy positivo para reconocer las principales falencias de estos puntos y la posibilidad de plantear propuestas para la transformación de los conflictos manifiestos.

Caracterización de la población atendida: de manera generalizada, este asunto fue el que cobró mayor importancia entre los equipos gestores, y más tiempo para su planteamiento, definición de criterios a interrogar, elaboración de herramientas para la recolección de la información, sistematización, análisis de resultados e interpretación. Para este propósito se concertaron los siguientes componentes: composición general de la población estudiantil, capacidades diversas, condiciones de excepcionalidad, estilos de aprendizaje y prácticas de enseñanza, intereses y expectativas, valoración de la educación, recursos y apoyos para el proceso educativo. En la actualización de este apartado, la actividad predominante fue la construcción de dos encuestas: una para padres de familia 
y otra para estudiantes, con el fin de obtener datos que orientaran la caracterización de estas dos poblaciones. Un asunto considerado muy problemático fue la tabulación de las encuestas, transcribirlas y ponerlas en tablas estadísticas, pues muchas veces esta labor se delega a profesores del área de matemáticas, considerándose como una sobrecarga laboral. Una alternativa fue la aplicación de la encuesta en línea, puesto que la recopilación de la información es más ágil, facilitando la organización de cifras estadísticas; esto sin contar con el ahorro de papel. En los grados de primaria, la encuesta se aplicó en físico para ser diligenciada con el apoyo de los padres, lo cual es muy útil en los grados de transición por su corta edad. El otro trabajo realizado fue la designación de uno o dos responsables de la elaboración de un documento descriptivo de caracterización por cada una de las sedes. El documento ya consolidado se revisó en las reuniones de acompañamiento y las últimas observaciones estuvieron a cargo de coordinadores y rectores, quienes presentaron el texto con las respectivas modificaciones.

\subsection{La propuesta pedagógica}

Los maestros de las diferentes IEO participantes, coincidieron en abordar este asunto teniendo en cuenta los planteamientos y herramientas aportadas en las sesiones del Diplomado destinadas a tal fin, por considerarlas prácticas y aterrizadas de la teoría a las realidades escolares, y referidas a aspectos puntuales de la propuesta pedagógica.

La revisión de los modelos pedagógicos reseñados en los PEI fue una de las tareas asumidas en las reuniones, en las que se reflexionó y cotejó el modelo pedagógico con las prácticas pedagógicas y los objetivos del modelo, en relación al horizonte institucional de cada IEO. Esto permitió un ajuste a los diversos planteamientos expresados en el documento y que, a consideración de los equipos de trabajo, fueron objeto de modificación. Esta reflexión conllevó a que la propuesta pedagógica recogiera no sólo lo que los docentes hacen diariamente en los procesos concretos que orientan, haciendo relación a lo interno de sus prácticas, sino también a los aspectos externos. Entre estos últimos se tienen: articulación con lineamientos MEN-PEI, competencias, estándares básicos de competencia y derechos básicos de aprendizaje, selección de contenidos, elección de recursos didácticos, relación pedagógica con la evaluación, usos pedagógicos de la evaluación, lineamientos y políticas nacionales e internacionales en el campo de la educación, indicadores de resultados, junto con las problemáticas de ausentismo, desmotivación y deserción escolar.

Adicionalmente, algunos componentes relevantes para el enfoque pedagógico, según el aporte de los maestros, implica considerar también: la estructura curricular de la escuela, el horizonte institucional, la caracterización de la IEO y las necesidades del entorno, que determinan gran parte de la denominada filosofía educativa institucional.

Herramientas para reflexionar sobre el Modelo Pedagógico: fue muy útil realizar un ejercicio de aproximación a las líneas pedagógicas que enmarcan la práctica de los maestros, para posteriormente reflexionar en torno a la pertinencia de apropiar un modelo que oriente su estructura curricular, en sintonía con su propósito educativo. Puede realizarse una encuesta aplicada a los maestros de transición, primaria, secundaria y media técnica. También es muy importante sensibilizar a los maestros sobre los estilos de aprendizaje, tanto de los estudiantes como de ellos mismos, pues esto permitirá una mayor justicia en el aula (González-Calixto, PatarroyoDurán \& Carreño-Bodensiek, 2017). Las encuestas sobre estilos de aprendizaje son muy variadas y abundantes en la red, incluso describen las formas en las que cada estilo de aprendizaje adquiere métodos y técnicas de formación académica (RuizMacías \& Duarte, 2018). Es necesaria su aplicación también para los maestros, dado que pueden reconocer que desde su propio estilo de manera 
inconsciente pueden hacer la planeación de sus clases. Por ejemplo, en el campo del lenguaje escrito, se sugiere incluir las relaciones con el entorno de las familias y los conocimientos previos de los estudiantes, para que los docentes diseñen estrategias atractivas de lectura y escritura, en relación con la vida social; estas habilidades no son solo actividades cognitivas, sino que son también herramientas culturales y sociales, como afirma Bernal-Pinzón (2017) al sugerir que "Los docentes deberían partir de la indagación en sus estudiantes de las creencias y elaboraciones personales acerca de la escritura, para potenciar, modificar y ampliar sus conocimientos".

Se abordó la construcción del Modelo Pedagógico desde los siguientes aspectos: los métodos de enseñanza-aprendizaje, las relaciones pedagógicas, las prácticas pedagógicas, tipos y usos de los recursos, los posibles estilos pedagógicos, Teorías del Desarrollo, Teorías del Aprendizaje, Teorías de las Disciplinas.

Otro insumo valioso para la reflexión de los equipos gestores, fue el texto: manejando Procesos Pedagógicos, bases para la enseñanza (Zaidiza-de Matos, 2006), puesto que describe la conceptualización de cada modelo pedagógico, sus características, ventajas, desventajas, formas de evaluación y recomendaciones para su desarrollo; un documento de este tipo ayuda a resolver en buena medida una necesidad manifiesta de los maestros y directivos sobre cómo aterrizar las teorías y postulados de los autores de referencia de este campo a sus realidades de manera sencilla, clara y directa. De este modo, facilitó la identificación en uno o varios de los modelos pedagógicos y coadyuvó a la reflexión de los docentes sobre sus prácticas y las herramientas necesarias para su perfeccionamiento.

\section{Conclusiones}

(...) El que aprende necesita comprender lo que hace (conciencia de las metas inmediatas aunque también las más o menos alejadas), conocer las razones que justifican la elección de las acciones seleccionadas para conseguir la meta (¿por qué se hace esto y no aquello?), comprender la organización de su desenvolvimiento (¿por qué se encadenan de esta manera los elementos que son o deben ser sucesivamente aprehendidos?), asegurar un nexo entre desarrollo y su propio devenir. Todo esto nos orienta hacia una pedagogía del proyecto: proyecto de uno mismo como respuesta a la necesidad de un marco del saber, y proyecto temático como respuesta a un marco organizador de las actividades para el estudio del objeto definido. (Nod, 1992)

Una Institución Educativa no puede ser pensada únicamente como la cuna de formación de los futuros ciudadanos del mundo, sino que debe explicitar en el presente la formación de los seres humanos que habitan el mundo local y global contemporáneo, pues es desde esos espacios, que debe nacer como un pilar fuerte el uso y aplicación del pensamiento crítico, para que los estudiantes puedan aprender a gestionar y usar desde el saber-hacer los sistemas de información integrados y robustecidos por toda la comunidad académica, para la generación y gestión de conocimiento que permita los desarrollos de aprendizajes colaborativos y acordes a las necesidades de nuestros entornos locales, nacionales y mundiales. Nuestros estudiantes ya no son ciudadanos de un sector o una comuna, son sujetos sociales mundiales que deben trabajar mancomunadamente con el resto del mundo en aspectos y necesidades globales como el medio ambiente, la paz mundial, la sostenibilidad del planeta, la democratización del conocimiento y el uso de los recursos naturales y la consolidación de la enorme riqueza cultural y social que tenemos para el mundo. Estos son algunos de los muchos aspectos que deben ser puestos dentro de los proyectos educativos institucionales, con el apoyo y uso de las herramientas tecnológicas, que le permitan a la comunidad educativa estar a la vanguardia de la generación de conocimientos y el 
desarrollo de hábitos y habilidades del ciudadano del siglo XXI.

En términos generales, se ha identificadoy valorado que los maestros tienen calificada experiencia profesional, formación académica e interés. Sin embargo, deseamos resaltar la continuidad a los procesos como una de las necesidades más sentidas por los docentes y las directivas de las IEO participantes, quienes en el primer encuentro con el equipo de trabajo, exponen su desencanto frente a este tipo de proyectos, dado que no hay garantía de continuidad. En palabras de los maestros: "se interesan, se cautivan y en el momento de mayor construcción y apropiación, finaliza la intervención". En algunos casos, con tareas pendientes, y en otros, con el deseo de profundizar más. Para los profesionales de acompañamiento comprender estas apreciaciones constituye uno de los principales desafíos para generar confianza y que se disponga con pertinencia y entusiasmo al trabajo del proyecto; un buen ejercicio para contrarrestar este sentir fue la elaboración de un plan de acción institucional, que ayudó a proyectar las posibilidades para su continuidad y mantener algunos espacios para la discusión pedagógica durante todo el año escolar.

En palabras de Fernández-Guerrero y GonzálezFierro (2017), podríamos finalizar afirmando que lo encontrado en los maestros hoy, a partir de sus saberes, haceres y sentires, demuestra que una parte de la comunidad académica: "viene realizando esfuerzos por dejar atrás el viejo esquema educativo, rígido en su estructura académica y basado en la transmisión de conocimientos, y está construyendo otro que sea un verdadero proceso dinamizador del desarrollo individual, cultural y social".

\section{Referencias}

Bernal-Pinzón, M.L. (2017) ¿qué escriben los niños?, una mirada desde el modelo escuela nueva. Revista de Investigación, Desarrollo e Innovación, 7 (2), 255268. doi: 10.19053/20278306.v7n2.2017.6069
Colmenares, A. (2012). Investigación-acción participativa: una metodología integradora del conocimiento y la acción. Voces y Silencios. Revista Latinoamericana de Educación, 3 (1), 102-115.

Ley 115. Congreso de la República. Por la cual se expide la ley general de educación. Bogotá, Colombia, febrero 8 de 1994.

Duran, A. (1994). El Proyecto Educativo Insitucional: una alternativa para el desarrollo pedagógicocultural. Bogotá, Colombia: Magisterio.

Fernández-Guerrero, M. M., \& González-Ferro, V. (2017). Enseñar, el arte de transformar y crecer. Saber, Ciencia y Libertad, 12 (2), 167-174. Recuperado de: http://www.sabercienciaylibertad. org/ojs/index.php/scyl/article/view/242

Garcés-Prettel, M., \& Ruiz-Cantillo, R. (2016). Integración pedagógica de la tecnología informática en instituciones educativas oficiales de Cartagena de indias (Colombia). Saber, Ciencia $Y$ Libertad, 11 (1), 175-186. doi: http://dx.doi. org/10.22525/sabcliber2016v11n1.184

García-Amaya, R. A., Fernández-Morales, F. H., \& Duarte, J. E. (2017). Modelo de integración de las TIC en instituciones educativas con características rurales. Revista Espacios, 38 (50), 26. Recuperado de: http://www.revistaespacios.com/ a17v38n50/17385026.html

García-Márquez, G. (1994). Por un país al alcance de los niños. Colombia al filo de la oportunidad. Bogotá, Colombia: Informe Conjunto de la Misión de Ciencia Educación y Desarrollo.

Gómez-Restrepo, C. et al., (2014). Conocimiento adquirido y adherencia en un diplomado sobre la salud mental para docentes orientadores. Revista de Salud Pública, 16, 821-833. doi: https://doi. org/10.15446/rsap.v16n6.51830 
González-Calixto, M., Patarroyo-Durán, N., \& Carreño-Bodensiek, C. (2017). El principio de justicia en el aula y responsabilidad moral del docente, frente a los estilos de aprendizaje. Revista de Investigación, Desarrollo e Innovación, 7 (2), 241-253. doi: https://doi.org/10.19053/20278306. v7.n2.2017.4497

Gutiérrez-Rodríguez, C. A. (2018). Fortalecimiento de las competencias de interpretación y solución de problemas mediante un entorno virtual de aprendizaje. Revista de Investigación, Desarrollo e Innovación, 8(2), 279-293. doi: 10.19053/20278306. v8.n2.2018.7170

Lozano, L. A. (1999). Paradigmas y tendencias de los Proyectos Educativos Institucionales. Bogotá, Colombia: Magisterio.

Marczak, J., Engelke, P., Bohl, D., \& SaldarriagaJiménez, A. (2016). América Latina y el Caribe 2030 - Escenarios Futuros. Atlantic Council: Biblioteca Felipe Herrera, Banco Interamericano de Desarrollo.

Ministerio de Educación Nacional, MEN. Decreto 1860. Por el cual se reglamenta parcialmente la Ley 115 de 1994, en los aspectos pedagógicos y organizativos generales. Bogotá, Colombia, agosto 3 de 1994.

Montes-Miranda, A. J. (2013). La educación básica en Colombia: una mirada a las políticas educativas. Saber, Ciencia y Libertad, 8 (2), 141-155. Recuperado de: http://www.sabercienciaylibertad.com/ojs/ index.php/scyl/article/view/106
Not, L. (1992). La enseñanza dialogante. Barcelona, España: Herder.

Ramírez, R., Caviedes, G., Gutiérrez, C., Vélez, W., Campo, F., \& Becerra, A. (2000). Referentes y Avances para la fundamentación del Proyecto Educativo Institucional. Cali, Colombia: Universidad del Valle.

Rodriguez. (2015). Veinte años de la Ley General de Educación: resultados y posibilidades. Bogotá, Colombia: Magisterio.

Ruiz-Macías, E., \& Duarte, J. (2018). Diseño de un material didáctico computarizado para la enseñanza de Oscilaciones y Ondas, a partir del estilo de aprendizaje de los estudiantes. Revista de Investigación, Desarrollo e Innovación, 8 (2), 295-309. doi: https://doi.org/10.19053/20278306. v8.n2.2018.7966

Trujillo, M. (comp.) (2017). Informe interno de acompañamiento al Fortalecimiento del PEI (inédito). Documento de trabajo construido a partir de los reportes de Mg. Ma. Cristina Hurtado Zúñiga, Mg. Francisco Medina, Mg. Marleny Ordoñez Olmedo, Mg. Paola González Pineda, Mg. Sol Ma. Gómez, Mg. Beatriz Grisales Herrera, Prof. Julieth Pérez Paredes, PhD. (c). Julio Cesar Sepúlveda, Lic. Inés Villanueva Tarquino.

UNESCO. (2014). Informe Anual. Chile: Oficina Regional de Educación para América y el Caribe.

Zaidiza-de Matos, B. (2006). Manejando procesos pedagógicos: bases para la enseñanza. Universidad Popular del Cesar. 
